\title{
Vertical Mandibular and Trunk Symmetry in Indonesian Orthodontic Patients
}

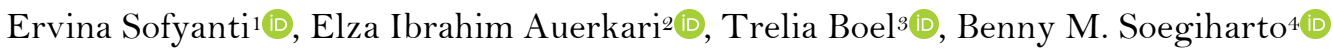

\begin{abstract}
'Department of Orthodontics, Faculty of Dentistry, Universitas Sumatera Utara, Medan, Indonesia.
2Department of Oral Biology, Faculty of Dentistry, Universitas Indonesia, Jakarta, Indonesia.

${ }^{3}$ Department of Dentomaxillofacial Radiography, Faculty of Dentistry, Universitas Sumatera Utara, Medan, Indonesia.

${ }^{4}$ Department of Orthodontics, Faculty of Dentistry, Universitas Indonesia, Jakarta, Indonesia.
\end{abstract}

Correspondence: Elza Ibrahim Auerkari, Department of Oral Biology, Faculty of Dentistry, Universitas Indonesia, Jl. Salemba Raya No. 4, Jakarta, Indonesia. E-mail: elza.ibrahim@ui.ac.id

Academic Editor: Alessandro Leite Cavalcanti

Received: 28 February 2020 / Review: 30 July 2020 / Accepted: 25 September 2020

How to cite: Sofyanti E, Auerkari EI, Boel T, Soegiharto BM. Vertical mandibular and trunk symmetry in Indonesian orthodontic patients. Pesqui Bras Odontopediatria Clín Integr. 202 1; 21:e5917. https://doi.org/10.1590/pboci.2021.066

\begin{abstract}
Objective: To analyze differences in vertical mandibular and trunk symmetry in orthodontic patients. Material and Methods: This was a cross-sectional study of 129 growing orthodontic patients who sought orthodontic treatment at the Dental Hospital Universitas Sumatera Utara, Indonesia. Mandibular symmetry index was observed with pre-treatment panoramic radiography based on Kjellberg's technique and trunk symmetry was evaluated based on questionnaires and visual observation. Vertical mandibular asymmetry was decided if the index of asymmetry was lower than $93.7 \%$. The bivariate analysis used the chi-squared and Fisher's exact tests, with a significance level of $5 \%$. Results: There was a significant association between vertical mandibular and trunk symmetry $(\mathrm{p}<0.05)$. The prevalence odds ratio for the association with vertical mandibular asymmetry was 3.007 (95\% CI $=1.016-8.905)$ for trunk asymmetry. Conclusion: The necessity to consider trunk symmetry could be included in orthodontics treatment of any malocclusion with vertical mandibular asymmetry that might require a multidisciplinary approach in the future.
\end{abstract}

Keywords: Orthodontics; Facial Asymmetry; Radiography, Panoramic. 


\section{Introduction}

Bilateral differences occur everywhere in vertebrae naturally and mark asymmetry in the anatomy of the body. Even though a small amount of asymmetry in the maxillofacial region is common, a critical threshold distance is considered clinically asymmetric [1-3]. In dentistry, the classification of asymmetries according to the involving structures includes dental, skeletal, muscular, and soft tissue and functional parameters. These slight facial asymmetries are acceptable esthetically. However, more significant asymmetry may cause functional as well as esthetic problems [1,3,4].

The etiology of mandibular asymmetry is vast and might be a combination of genetic and environmental influences. The causes of mandibular asymmetry can be stratified as developmental, pathological, traumatic, and functional [4,5]. Some previous studies have hypothesized that postural disorders are involved in the stomatognathic system [6-8]. A similar theory about the development of the craniocervical complex based on individual muscular balances [9] associated with regional imbalances has been deemed a contributory factor in facial asymmetry and shoulder imbalance in adolescent subjects. Morphological features of the odontoid process may serve as valuable predictive markers in interdisciplinary orthopedic-orthodontic diagnostics $[10,11]$.

Considering the role of the myofascial system associated with TMD, occlusal changes, and tooth loss have wisely been recommended in any muscular-skeletal disorder treatment. This, related to postural alterations, may reflect a general lack of equilibrium in the individual [6]. According to Alghadir et al. [12], jaw clenching can enhance postural stability during standing on an unstable surface in both conditions of the presence and absence of visual input in healthy adults. In treatment and rehabilitation planning for patients, postural instability should be considered. Segatto et al. [13] suggested that mandibular asymmetry measurements could substitute disease-marker measurements obtained during the evaluation of the lateral cephalograms and, similarly to rasterstereography, further reduce the radiation load of the involved orthopedic subjects $[12,13]$.

The mystery of asymmetry was still unsolved due to developmental facial asymmetry that is idiopathic and gradually develops over the years after birth, which might become prominent during the adolescent period. In young adults, visual perception control is most important in orienting the head in the frontal plane and related to the standing surface and postural control in adaptation efforts to maintain the head position [14]. Otherwise, the explanations of why most asymmetries cannot be treated with single-jaw surgery include occlusal canting in the planning and management of dentofacial deformities of the complexity of some malocclusions related to imbalanced mandibular function and development.

Since some previous studies have reported on the growth and development of skeletal postural and mandibular aspects in a similar period, the objective of this study was to analyze the differences in vertical mandibular and trunk symmetry in young adult subjects who sought orthodontic treatment at the Dental Hospital Universitas Sumatera Utara, Medan, Indonesia.

\section{Material and Methods}

Study Design and Ethical Clearance

This cross-sectional study was conducted at the Dental Hospital Universitas Sumatera Utara between June 2016 and March 2017 and was approved by the Health Research Ethical Committee of the Universitas Sumatera Utara Medical Faculty (100/DATE/KEPK FK USU-RSUP HAM/2017). In compliance with the 
Declaration of Helsinki, subjects who consented as participants were included in a survey and provided written informed consent.

Sample and Data Collection

Patients aged between 11 and 25 years old who willing to participate in this study were included. Participants had no history of congenital disease or previous orthodontics treatment. The mandibular symmetry index was analyzed based on Kjellberg's technique using the pre-treatment panoramic radiograph $[15,16]$. A trunk symmetry assessment was conducted using the visual observation method and by answering the modified questionnaires under spinal orthopedic surgeon supervision [17,18] (Table 1). If any of the questions in the modified questionnaires and visual observation method of trunk symmetry assessment was positive, it was categorized as suggesting trunk asymmetry. Vertical mandibular asymmetry was decided if the asymmetry index was lower than $93.7 \%$ (Figure 1).

Table 1. Modified questionnaires and visual observation method of trunk symmetry.

\begin{tabular}{l} 
No. Questionnaires related to trunk symmetry assessment (position while standing Category \\
straight in front of the mirror) \\
\hline 1. \\
Are your left and right shoulders at the same height? \\
3. Is either side of your shoulders more forward than the other side? \\
4. Are your left and right ears at the same level? \\
Are your left and right hips on the same level when standing in an upright position? \\
Are the left and right of your sleeves of your right and left arms at the same level \\
Are the left and right legs of your pants at the same height on both sides when \\
wearing tight pants?
\end{tabular}
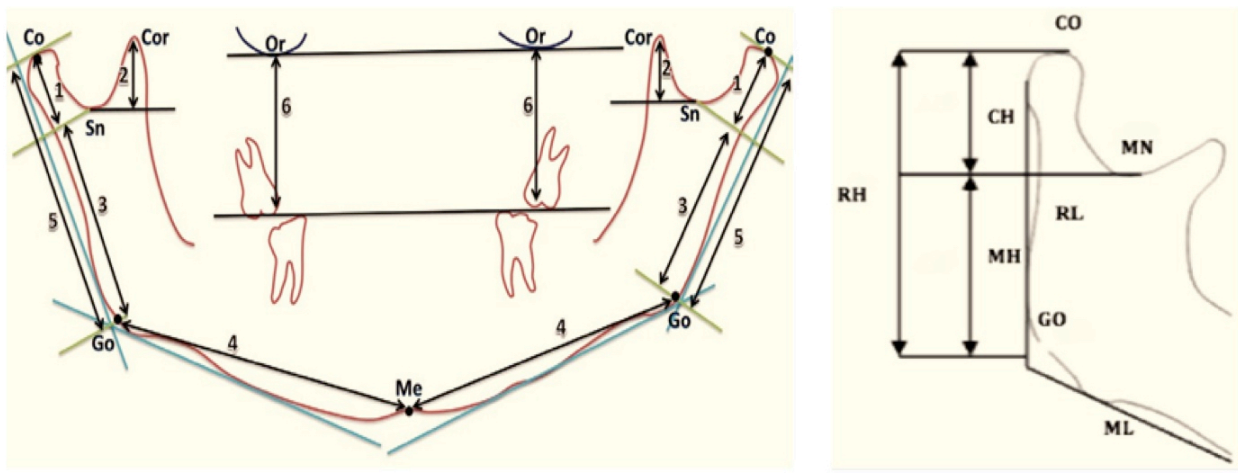

Figure 1. Vertical mandibular symmetry determined based on Kjellberg's technique.

Data Analysis 
The significance of association between the trunk and vertical mandibular symmetry was evaluated using the chi-squared and Fisher's exact tests, with assumed significance at $p<0.05$. All statistical analyses were performed using the Statistical Package for the Social Sciences version 18.0 software program (IBM Corp., Armonk, NY, USA) [19].

\section{Results}

The validity and reliability vertical mandibular symmetry used 20 panoramic radiographs measured using Cohen's $\kappa$ and displayed moderate agreement for inter-rater measurements $(\kappa=0.538)$ between a dentomaxillofacial radiologist and orthodontist and intra-rater measurements $(\kappa=0.674)$ as conducted by an orthodontist. The modified questionnaires' validity during trunk symmetry assessment by Pearson correlation coefficient showed a critical value of $r=0.422(\mathrm{ES}$ and $\mathrm{TB})$. The reliability of the modified questionnaires used in trunk symmetry assessment by Cronbach's alpha was $0.800(\mathrm{r} \geq 0.6)$. There were 129 growing orthodontics subjects (mean age: $20.7 \pm 3.2$ years old) who attended the Dental Hospital Universitas Sumatera Utara involved in this cross-sectional study. Among these studied subjects, Table 2 indicates that there was no significant difference ( $\mathrm{p}>0.05)$ of vertical mandibular symmetry between early adolescents (11-18 years) and late adolescents (19-25 years).

Table 2. Characteristics of age-based vertical mandibular symmetry.

\begin{tabular}{cccc}
\multirow{2}{*}{ Age } & \multicolumn{2}{c}{ Vertical Mandibular Symmetry } & \\
& Symmetry & Asymmetry & p-value \\
& $\mathrm{N}(\%)$ & $\mathrm{N}(\%)$ & \\
\hline Early Adolescence & $7(5.42)$ & $22(17.05)$ & 0.136 \\
Late Adolescence & $43(33.33)$ & $57(44.18)$ & \\
\hline
\end{tabular}

Table 3 presents that $34.88 \%$ of the total study population had trunk symmetry and $3.88 \%$ had trunk asymmetry. Broken down further, $43.41 \%$ of the study population with vertical mandibular asymmetry had trunk symmetry and $17.83 \%$ had trunk asymmetry. There was thus a significant difference $(p=0.047)$ in trunk symmetry based on vertical mandibular symmetry. The final multivariate analysis model using the backward stepwise method showed that the prevalence odds ratio for the association with vertical mandibular asymmetry was 3.007 (95\% CI: 1.016-8.905) for trunk asymmetry.

Table 3. Characteristics of trunk symmetry based on vertical mandibular symmetry.

\begin{tabular}{ccccc}
\hline \multirow{2}{*}{ Trunk } & \multicolumn{2}{c}{ Vertical Mandibular Symmetry } & & \\
& Symmetry & Asymmetry & p-value & Odds Ratio \\
& $\mathrm{N}(\%)$ & $\mathrm{N}(\%)$ & & \\
\hline Symmetry & $45(34.88)$ & $56(43.41)$ & $0.047^{*}$ & 3.007 \\
Asymmetry & $5(3.88)$ & $23(17.83)$ & & $(1.016-8.905)$ \\
\hline *Statistically Significant. & & &
\end{tabular}

*Statistically Significant.

\section{Discussion}

This study sought to analyze the difference in vertical mandibular and trunk symmetry in orthodontic patients. Even though abnormal body posture has long been hypothesized as responsible for various malocclusions and dentofacial deformity development, some literature still does not support these assumptions. Although facial asymmetry and malocclusion are treated as focal pathological states, these deformities can originate from a faulty posture of the trunk [11]. 
Previous studies on idiopathic scoliosis have been mainly focused on examining abnormal body posture as it relates to malocclusion, facial asymmetry, and skeletal problems due to issues with balancing of the stomatognathic system [9-11,13]. In this study, vertical mandibular asymmetry was $85.69 \% \pm 6.48 \%$, whilst the asymmetry was $97.15 \% \pm 1.97 \%$ based on the Kjellberg technique. There was no significant difference between early adolescents and late adolescents ( $\mathrm{p}>0.05)$ despite late adolescent walk-in patients being more prevalent than early adolescents in this cross-sectional study (Table 2). However, one must consider the skeletal age in addition to the chronological age while treating any malocclusion with asymmetry.

Table 3 indicates that the prevalence odds ratio for the association with vertical mandibular asymmetry was 3.007 (95\% CI: 1.016-8.905) for trunk asymmetry. The hypothesis of compensatory spine curvature inducement might lead to compensatory head posture in three dimensions. Then, the phenotype of asymmetric posture can be used to help with early detection to avoid complex orthodontic treatment related to malocclusion with mandibular asymmetry.

Early identification of developmental skeletal asymmetry to obtain homeostasis in vertebrae, associated with fluctuating asymmetry derived from deviations in ideal symmetry, is possible. This functions as a bioindicator to reflect the level of adaptive genetic and environmental stress experienced by individuals or populations [3,15]. Observation of the development of mandibular asymmetry as related to mandibular condyle suggested that postural balance and occlusion pertaining to the adaptation process in the stomatognathic system include tooth, muscles, and joints [6-8].

The significant relationship of our finding in Table 3 was also supported by Harila et al. [20] that suggested asymmetric growth of occlusion and the development of crossbite are commonly found in children with congenital hip dislocation. A correlation was noted between mandibular deviation and shoulder imbalance related to the degree of scoliosis in adult subjects, presenting a linear trend [21]. Thus, the presence of mandibular and postural asymmetry in vertical and transversal assessments showed reciprocal relationships. These deformities should be clinically evaluated in the management of mandibular deviation [11,13,22]. The phenomenon of head position adaptation due to the shift of the mandible was identical to the phenomenon of body posture compensation in maintaining the balance function. The temporomandibular joint as a center growth point of the mandibular area affects body posture relative to other craniofacial parameters.

The evaluation of body posture is a special investigation parameter nowadays since the goal of orthodontic treatment includes achieving good stability in addition to aesthetics and function. Even though Algadhir et al. [12] suggested that jaw positions should be considered in treatment and rehabilitation planning for healthy patients with postural instability, a different study reported postural parameters found not to be accurate in the juvenile population with unilateral posterior crossbite [23].

The present study suggested how, overall, the body can compensate for this asymmetry phenomenon. However, our results differed from those of Arienti's study that stated trunk and facial asymmetry only based on clinical examinations were not associated with one another in the cross-sectional study of 1,029 healthy adolescents in Italy. Also, there was no apparent relation between the severity of scoliosis based on a coronal view of the spine and facial form variations based on skeletal type in idiopathic female scoliosis patients in Korea [24]. There was plausible evidence supporting an increased prevalence of unilateral angle class II malocclusions, lateral crossbite risk, and midline deviation in children affected by scoliosis [3]. Hong et al. [10] suggested that ramus length difference of right and left that reflected midsagittal line showed a possible correlation with the coracoid height difference, clavicular angle, radiographic shoulder height, and clavicle-rib intersection difference based on frontal cephalometric data $(\mathrm{p}<0.05)$. 
In understanding the growth and development of malocclusion subjects, scientific validation to date the development of mandibular asymmetry will help in diagnosing sociodemographic complexity. Information about the morphological aspect related to the measurement and degree of mandibular symmetry will help dental clinicians diagnose malocclusion to form a comprehensive treatment plan [12,21,25].

In a clinical setting, body posture could be considered when dental occlusion is developed. Vertical mandibular asymmetry closely related to the temporomandibular joint could determine the adaption of balancing the stomatognathic system through constant force of the cervical to spinal area. Mandibular shifts characterized by midline differences between the maxilla and mandible have a close relationship with the postural disorder [13]. In a clinical setting, body posture should be taken into account when dental occlusion is developing $[6-8,12,26]$. The assessment of asymmetrical phenotype in the trunk and facial symmetry should consider three-dimensional mandibular growth and development.

Since the complexity of asymmetry is not just evaluated in the dentomaxillofacial areas but also the whole body, future studies should conduct a comprehensive analysis, such as examining locomotor organs, due to the developmental process of asymmetry. However, the present early detection of asymmetry symptoms by questionnaires that has never before been done in any previous studies indicated that asymmetry should be considered. Longitudinal investigations will be required to achieve stability of the stomatognathic system. Since the stomatognathic system is a complex issue nowadays, further study should consider skeletal malocclusion variance, which is related to the adaptation process.

\section{Conclusion}

The bilateral symmetry of the mandibular area might influence the body equilibrium and vice versa in treatment malocclusion with mandibular asymmetry by panoramic radiograph analysis for early orthodontic and orthopedic prevention. The necessity to consider trunk symmetry could be advised during orthodontics treatment of any malocclusion with vertical mandibular asymmetry that might require a multidisciplinary approach in the future.

\section{Authors' Contributions}

\begin{tabular}{|c|c|c|}
\hline ES & (i) https://orcid.org/0000-0002-0144-4397 & $\begin{array}{l}\text { Conceptualization, Methodology, Investigation, Resources, Data Curation, Writing - Original } \\
\text { Draft, Writing - Review and Editing and Visualization. }\end{array}$ \\
\hline EIA & https://orcid.org/0000-0002-5680-7925 & Conceptualization, Writing - Review and Editing, Supervision and Project Administration. \\
\hline ТВ & (iD) https://orcid.org/0000-0002-8369-7696 & Validation, Formal Analysis, Data Curation and Visualization. \\
\hline BMS & (iD) https://orcid.org/0000-0003-0310-0990 & Methodology and Supervision. \\
\hline
\end{tabular}

\section{Financial Support}

This study was partially granted by the Ministry of Research, Technology and Higher Education through DRPM grant no. 116/UN5.2.3.1.PPP/KP-DRPM/2019 and 01410/UN2.R3.1.HKP.05.00/2019.

\section{Conflict of Interest}

The authors declare no conflicts of interest.

\section{Data Availability}

The data used to support the findings of this study can be made available upon request to the corresponding author.

\section{Acknowledgment}


We want to thank all participants of this study. Dr. Otman Siregar, MD (consultant spinal orthopedic surgeons) Derek Diong, Tommy Kwan, and Putri Eyanoer.

\section{References}

[1] Cheong Y, Lo L. Facial asymmetry: etiology, evaluation, and management. Chang Gung Med J 201 1; 34(4):341-51.

[2] Anison JJ, Rajasekar L, Ragavendra B. Understanding asymmetry - a review. Biomed Pharmacol J 2015; 8:659-68. https://doi.org/10.13005/bpj/764

[3] Thiesen G, Gribel BF, Freitas MPM. Facial asymmetry: a current review. Dental Press J Orthod 2015; 20(6):110-25. https://doi.org/10.1590/2177-6709.20.6.110-125.sar

[4] Maheswari S, Verma SK, Gaur A, Dhiman S. Diagnosis and management of facial asymmetries. J Orthod Res 2015; $3(2): 81-7$

[5] Chia MS, Naini FB, Gill DS. The aetiology, diagnosis and management of mandibular asymmetry. Orthod Updat 2008; 1(2):44-52. https://doi.org/10.12968/ortu.2008.1.2.44

[6] Cuccia A, Caradonna C. The relationship between the stomatognathic system and body posture. Clinics 2009; 64(1):61-6. https://doi.org/10.1590/s1807-59322009000100011

[7] Khan MT, Verma SK, Maheshwari S, Zahid SN, Chaudhary PK. Neuromuscular dentistry: Occlusal diseases and posture. J Oral Biol Craniofac Res 2013; 3(3):146-50. https://doi.org/10.1016/j.jobcr.2013.03.003

[8] Salkar RG, Radke UM, Deshmukh SP, Radke PM. Relationship between temporomandibular. Int J Dent Health Sci 2015; 2(6):1523-30.

[9] Saccucci M, Tettamanti L, Mummolo S, Polimeni A, Festa F, Tecco S. Scoliosis and dental occlusion: A review of the literature. Scoliosis 2011; 6:15. https://doi.org/10.1186/1748-7161-6-15

[10] Hong J, Suh S, Modi HN, Yang J, Hwang Y, Lee D, et al. Correlation between facial asymmetry, shoulder imbalance, and adolescent idiopathic scoliosis. Orthopedics 2011;34(6):187. https://doi.org/ 10.3928/01477447-20110427-14

[11] Segatto E, Segatto A, Braunitzer G, Kirschneck C, Fanghänel J, Danesh G, et al. Craniofacial and cervical morphology related to sagittal spinal posture in children and adolescents. Biomed Res Int 2014; 2014:638238. https://doi.org/10.1155/2014/638238

[12] Alghadir AH, Zafar H, Iqbal ZA. Effect of three different jaw positions on postural stability during standing. Funct Neurol 2015; 30(1):53-7.

[13] Segatto E, Lippold C, Végh A. Craniofacial features of children with spinal deformities. BMC Musculoskelet Disord 2008; 9:169. https://doi.org/10.1186/1471-2474-9-169

[14] Pociask FD, DiZazzo-Miller R, Goldberg A, Adamo DE. Contribution of head position, standing surface, and vision to postural control in community-dwelling older adults. Am J Occup Ther 2016; 70(1):7001270010p1-8. https://doi.org/10.5014/ajot.2016.015727

[15] Iturriaga V, Navarro P, Cantin M, Fuentes R. Prevalence of vertical condilar asymmetry of the temporomandibular joint in patients with signs and symptoms of temporomandibular disorders. Int J Morphol 2012; 30(1):315-21. https://doi.org/10.4067/S0717-95022012000100056

[16] Hirpara N, Jain S, Hirpara VS, Punyani PR. Comparative assessment of vertical facial asymmetry using posteroanterior cephalogram and orthopantomogram. J Biomed Sci 2017; 6(1):1-7. https://doi.org/10.21767/2254-609X.100052

[17] Singla D, Veqar Z. Methods of postural assessment used for sports persons. J Clin Diagn Res 2014; 8(4):LE01-4. https://doi.org/10.7860/JCDR/2014/6836.4266

[18] Adobor RD, Riise RB, Sørensen R, Kibsgård TJ, Steen H, Brox JI. Scoliosis detection , patient characteristics , referral patterns and treatment in the absence of a screening program in Norway. Scoliosis 2012; 7(1):18. https://doi.org/10.1186/1748-7161-7-18

[19] Coster GD, Dongen SV, Malaki P, Muchane M, Alcántara-Exposito A, Matheve H, et al. Fluctuating asymmetry and environmental stress: understanding the role of trait history. PLoS One 2013; 8(3):e57966. https://doi.org/10.1371/journal.pone.0057966

[20] Harila V, Valkama M, Sato K, Tolleson S, Hanis S, Kau CH, et al. Occlusal asymmetries in children with congenital hip dislocation. Eur J Orthod 2012; 34(3):307-11. https://doi.org/10.1093/ejo/cjroo4

[21] Zhou S, Yan J, Da H, Yang Y, Wang N, Wang W, et al. A correlational study of scoliosis and trunk balance in adult patients with mandibular deviation. PLoS One 2013; 8(3):e59929. https://doi.org/10.1371/journal.pone.0059929

[22] Lippold C, Moiseenko T, Drerup B, Schilgen M, Végh A, Danesh G. Spine deviations and orthodontic treatment of asymmetric malocclusions in children. BMC Musculoskelet Disord 2012; 13:151.

https://doi.org/10.1186/1471-2474-13-151

[23] Kim T, Kim J, Kim Y, Cho I, Lim Y, Lee D. The relation between idiopathic scoliosis and the frontal and lateral facial form. Korean J Orthod 2014; 44(5):254-62. https://doi.org/10.4041/kjod.2014.44.5.254

[24] Elslande DCV, Russett SJ, Major PW, Flores-Mir C. Mandibular asymmetry diagnosis with panoramic imaging. Am J Orthod Dentofacial Orthop 2008; 134(2):183-92. https://doi.org/10.1016/j.ajodo.2007.07.021

[25] Kasimoglu Y, Tuna EB, Rahimi B, Marsan G, Gencay K. Condylar asymmetry in different occlusion types. Cranio 2015; 33(1):10-4. https://doi.org/10.1179/0886963414Z.00000000039 
[26] Silvestrini-Biavati A, Migliorati M, Demarziani E, Tecco S, Silvestrini-Biavati P, Polimeni A, et al. Clinical association between teeth malocclusions, Wrong posture and ocular convergence disorders: An epidemiological investigation on primary school children. BMC Pediatr 2013; 13:12. https://doi.org/10.1186/1471-2431-13-12 